\title{
EXPERTENSEMINAR DAVOS 2016
}

$\mathrm{V}$

om 22.-26. Februar 2016 fand der traditionelle Expertenkurs in Davos zum zweiten Mal unter der Ägide der SACAM (Schweizerische Ärztegesellschaft für Akupunktur, Chinesische Medizin und Aurikulomedizin) statt. Erfreulich war, dass die Teilnehmerzahl seit letztem Jahr gewachsen ist. So fanden sich dieses Jahr fast 100 Teilnehmer im schönen Kursraum des Hotels Sunstar ein. Zum Einstimmen und als erstes Highlight fand zudem am Wochenende ein Vorkurs mit Prof. Bahr und Dr. Ramme unter dem Titel „Klassische energetische Akupunktur unter besonderer Berücksichtigung des Lasers" statt.

Das Eröffnungsreferat hielt Prof. Bahr mit der neuen Technik des „Ultimativen-Feuer-Schürens“, um äußerst effizient negatives Qi auszuleiten. Die dabei verwendeten Silber-Blockadepunkte ermöglichen eine neue Tiefe der Behandlung. Weitere Schwerpunkte von Prof. Bahr waren die Traumatherapie mittels Akupunktur auf dem Lenkergefäß und entsprechende Blütengabe, die Konstitutionstherapie und die energetische Anregung mittels Laserlicht. Prof. Wojak referierte über Herzrhythmusflexibilität als Indikator für die evidenzbasierte Akupunktur. Dr. Ramme stellte uns die „Liquidakupunktur“ vor, welche einen einfachen, aber gleichzeitig hoch wirksamen Einstieg in die Ohrakupunktur ermöglicht.

Den Österreicher Tag gestalteten die Kollegen aus dem Nachbarland mit einem bunten Programm: Dr. Reininger referierte über „Neue Aspekte der Nogier-Frequenzen“. Weitere Themen waren die Behandlung von Karzinomen und der MS mithilfe aurikulomedizinischer Techniken, die Behandlung von Potenz- und Libidostörungen in der TCM, eine Übersicht über die Extrapunkte sowie ein Einblick in die therapeutische Anwendung unserer heimischen Alpenblüten.

Prof.Wirz stellte die neue „Laseruhr“ vor, welche eine Heimbehandlung der Patienten ermöglicht sowie die von ihm entwickelte „Transflorale Akupunktur“ am Beispiel von Zahnstörfeldern. Ein umfassendes Behandlungskonzept der Makuladegeneration wurde von Dr. Kreisel vorgestellt. Howard Austin gab einen erfrischenden Einblick die Antlitzdiagnostik der TCM.

Weitere Beiträge zur Mitochondropathie, der Behandlung chronischer Hautkrankheiten mittels chinesischer Arzneimittel, der Buteyko-Atmung und zu verschiedenen Ernährungsweisen aus Sicht der RAC-Diagnostik rundeten das Bild ab.

Die bereits letztes Jahr eingeführten und beliebten Workshops fanden wieder statt. Die Qi-Gong-Liebhaber kamen reichlich auf ihre Kosten: Mit Meister Yaw Hwa Chin und Howard Austin hatten wir gleich zwei versierte Lehrer im Programm. Weitere Themen waren ein Kalligraphiekurs, eine Einführung in die Implantatakupunktur sowie ein Laser-Workshop. Derjenige, den es am Nachmittag nicht bei Sonnenschein auf die Skipiste zog, hatte also ein vielfältiges Programm.

Ein Höhepunkt kultureller Art war ein Konzert mit der klassischen chinesischen Kniegeige. Frau Xiang Alina führte uns gekonnt und mitreißend in chinesische Klangwelten, für unser westliches Ohr faszinierende und fremd anmutende Harmonien.

Daneben kam natürlich der informelle Teil nicht zu kurz: das abendliche gemütliche Zusammensein und der ungezwungene kollegiale Austausch in lockerer Atmosphäre.

Das nächste Expertenseminar findet vom 20.-24. Februar 2017 statt. Wir freuen uns auf ein Wiedersehen!

Das Organisationskomitee

Prof.VRC Andreas Wirz-Ridolfi

Dr. Thomas Holzknecht

Dr. Christoph Scholtes

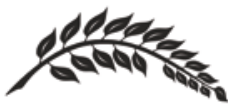

Wir trauern um

Frau Marianne Haase/Zahnärztin, Lübeck Herrn Wladimir Pawlow/Arzt, Lippstadt

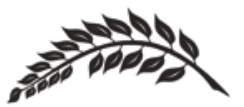

